\title{
Critical appraisal of adalimumab in the treatment of chronic plaque psoriasis
}

\author{
This article was published in the following Dove Press journal: \\ Psoriasis: Targets and Therapy \\ 24 June 2014 \\ Number of times this article has been viewed
}

\author{
Danielle Nicolazzo' \\ Franz Kerdel' \\ Francisco Kerdel ${ }^{1,2}$ \\ 'Florida Academic Dermatology \\ Centers, The University of Miami \\ Hospital, Miami, FL, USA; ${ }^{2}$ Florida \\ International University, Miami, \\ FL, USA
}

Correspondence: Francisco Kerdel Florida Academic Dermatology Centers, The University of Miami Hospital, I400 NW 12th Avenue, Suite 4, Miami, FL 33 I 36, USA

$\mathrm{Tel}+\mathrm{I} 3053242110$

Fax + I 3053250919

Email dermatology@fadcenter.com

\begin{abstract}
Psoriasis is a chronic inflammatory disease estimated to affect $1 \%-3 \%$ of the worldwide population. It is not simply a cutaneous disease but also poses a significant medical, social, and economic burden worldwide. Tumor necrosis factor (TNF)- $\alpha$ inhibitors are currently amongst the most important drugs in the therapeutic management of psoriasis. Patients using TNF- $\alpha$ inhibitors are at risk for infections including active and latent tuberculosis. Adalimumab, one of the TNF- $\alpha$ inhibitors, is a fully human monoclonal antibody that received approval for the treatment of chronic plaque psoriasis in 2008. Its use has been studied extensively for its safety and efficacy profile in the clinical setting. For the purpose of this review, we accessed the major publications in English relating to the use of adalimumab for psoriasis. Adalimumab appears to be one of the most efficacious biologic agents for the treatment of psoriasis. Results from various clinical trials including the REVEAL, CHAMPION, and BELIEVE are included in this review. The most recent data from an ongoing post-marketing study (ESPRIT) is also included in the analysis. Research regarding the safety, efficacy, and patient-reported outcomes support a favorable risk-benefit profile for adalimumab.
\end{abstract}

Keywords: TNF- $\alpha$ inhibitor, safety, efficacy, outcomes

\section{Introduction}

Psoriasis is a chronic inflammatory disease estimated to affect $1 \%-3 \%$ of the worldwide population, with reports as high as $4.6 \%$ in the US. ${ }^{1}$ While an exact genetic abnormality has not been identified, psoriasis occurs with increased frequency in families. A polygenic inheritance of HLA (human leukocyte antigen) subtypes along with endogenous and exogenous triggers predisposes individuals to developing the disease. ${ }^{2}$ There are currently five types of psoriasis separated by morphology (chronic plaque, guttate, erythrodermic, pustular, and inverse), with chronic plaque psoriasis accounting for more than $90 \%$ of cases. Psoriasis is not simply a cutaneous disease; it poses a substantial medical, social, and economic burden. Studies suggest that patients with psoriasis have a decreased quality of life and are at an increased risk of heart disease, depression, metabolic syndrome, diabetes, and some malignancies (ie, lymphoma). ${ }^{3,4}$ The financial costs associated with direct medical care for psoriasis in the US is estimated at US\$11.25 billion annually. ${ }^{5}$

Treatment for psoriasis has evolved over the years as our understanding of the pathogenesis continues to expand. Treatment is guided by the extent of disease, with the majority of patients being controlled with topicals and phototherapy. For more extensive disease, systemic therapy is required. Prior to the $1980 \mathrm{~s}$, biochemical mediators, enzymes, and pathways that were proven to be abnormal in psoriasis guided treatment. 
It was not until T-cell suppressive agents, such as cyclosporine, demonstrated superior clinical results that psoriasis became regarded as a T-cell mediated disease. ${ }^{6,7}$

More specifically, psoriasis is a T-cell disease as evidenced by an increased amount of the T helper (Th) cell cytokines in lesional skin. Langerhans and dendritic cells are also present in psoriatic skin and have immunostimulatory effects, leading to further activation of Th cells. The release of interleukin (IL)-23 and IL-12 by dendritic cells leads to the differentiation of naïve T-cells into Th-1 cells and Th-17 cells, and also stimulates the production of interferon (IFN)- $\gamma$ and tumor necrosis factor (TNF)- $\alpha$ from natural killer cells. ${ }^{1}$ It is this increase in TNF- $\alpha$, IFN- $\gamma$, and other ILs that leads to keratinocyte proliferation, the formation of psoriatic plaques, and increased inflammation. In the past several years, pharmaceutical companies have focused their treatment modalities on targeting these cytokines in an attempt to achieve the greatest therapeutic response. Several biologics have surfaced as potential therapies for the treatment of psoriasis, including the IL-12 and IL-23 monoclonal antibody ustekinumab, the CD2 (cluster of differentiation 2) targeting alefacept (removed from the market), and multiple TNF- $\alpha$ inhibitors. Inhibition of IL-17 (subtypes A and F) or its receptor shows promise and is currently under investigation.

TNF- $\alpha$ is a transmembrane protein that belongs to the TNF superfamily, which is comprised of at least 20 proteins. It is a homotrimer, proinflammatory cytokine with a wide diversity of cellular activities. Numerous experimental and clinical investigations have shown increased levels of TNF- $\alpha$ in lesional psoriatic skin, increased mRNA (messenger ribonucleic acid) expression in peripheral blood mononuclear cells of psoriatic patients and increased plasma concentration levels. Two receptors for TNF- $\alpha$ have been identified - p55 (TNF receptor [TNFR]-1), and p75 (TNFR-2). Interestingly, differential expression of TNFR-1 and TNFR-2 has been demonstrated in uninvolved versus psoriatic skin of psoriasis patients. $^{8}$

TNF- $\alpha$ inhibitors are currently amongst the most important drugs in the therapeutic management of psoriasis. ${ }^{9}$ There are currently five TNF- $\alpha$ inhibitors available, and only three of them are US Food and Drug Administration (FDA) approved for treating psoriasis: adalimumab, a fully human monoclonal antibody; etanercept, a soluble receptor construct; and infliximab, a chimeric monoclonal antibody. ${ }^{8}$ The other two - golimumab, a fully human monoclonal antibody, and certolizumab, PEGylated Fab fragment of a humanized TNF- $\alpha$ inhibitor monoclonal antibody - are FDA approved for psoriatic arthritis but not psoriasis.
In the US, adalimumab is indicated for use in rheumatoid arthritis, ankylosing spondylitis, inflammatory bowel disease, psoriatic arthritis, and juvenile idiopathic arthritis and was approved for the treatment of plaque psoriasis in 2008. It binds specifically to TNF- $\alpha$, not to TNF- $\beta$, and blocks its interaction with p55 and p75 soluble and membrane-bound receptors. It was originally developed by BASF Pharma (Ludwigshafen, Germany) to be structurally and functionally indistinguishable from the naturally occurring human immunoglobulin G1 antibody. In terms of pharmacokinetics, the average absolute bioavailability is $64 \%$ after a single $40 \mathrm{mg}$ dose. The half-life is 14 days, which leads to every other week dosing. ${ }^{10}$

\section{Methods}

For the purpose of this review, we accessed the major publications in English relating to the use of adalimumab for psoriasis. We excluded studies specifically targeting psoriatic arthritis as well as case reports. For the purpose of assessing post-marketing data, we reviewed the ESPRIT registry, which is an ongoing 10-year observational registry of adalimumab in patients with chronic plaque psoriasis. Most studies reported primary outcomes as PASI-75 (percentage of patients improving by at least $75 \%$ ) between 12 and 16 weeks from initiation of therapy. Secondary outcomes such as PASI-50, PASI-90, and patient-related outcomes, such as quality of life measures, were also obtained from these articles.

\section{Results Efficacy}

Adalimumab appears to be one of the most efficacious biologic agents for the treatment of psoriasis. In a Phase II randomized, double-blinded, placebo-controlled study, 147 patients received either adalimumab $40 \mathrm{mg} /$ week versus $40 \mathrm{mg}$ every other week (eow) versus placebo. At week 12, $53 \%$ of eow and $80 \%$ of weekly achieved PASI-75. Only $4 \%$ of patients on placebo achieved this result. After 12 weeks, patients were allowed to continue their assigned doses for a 48-week extension; patients on placebo were switched to 40 mg eow. Responses were sustained during the open extension, and the drug appeared to be well tolerated. ${ }^{11}$

The REVEAL (Randomized Controlled Evaluation of Adalimumab) study (Table 1) was a Phase III randomized controlled evaluation of adalimumab every other week dosing in moderate to severe psoriasis. ${ }^{12}$ The study design was divided into three periods: A, B, and C. Periods A and C were double-blind, placebo-controlled, while period B was open-label. Eligibility criteria included adult patients with 
Table I A comparison of three Phase III clinical trials on the use of adalimumab for treating chronic plaque psoriasis

\begin{tabular}{|c|c|c|}
\hline Study & Study design & Treatment duration and regimen \\
\hline $\begin{array}{l}\text { REVEAL }^{a} \\
(n=I, 2 \mid 2)\end{array}$ & $\begin{array}{l}\text { Phase III randomized controlled study of } \\
\text { adalimumab every-other-week dosing in } \\
\text { patients with moderate to severe plaque } \\
\text { psoriasis }\end{array}$ & 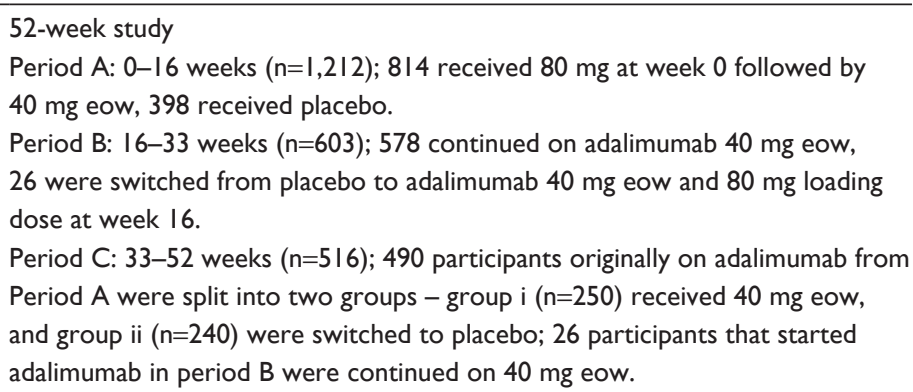 \\
\hline $\begin{array}{l}\text { CHAMPION }^{b} \\
(n=27 \mathrm{I})\end{array}$ & $\begin{array}{l}\text { Phase III randomized, double-blind, } \\
\text { placebo-controlled study of the efficacy of } \\
\text { adalimumab vs methotrexate vs placebo }\end{array}$ & $\begin{array}{l}\text { I6-week study } \\
\text { Adalimumab group: }(\mathrm{n}=108) \text {, administered } 80 \mathrm{mg} \text { loading dose, then } 40 \mathrm{mg} \text { eow } \\
\text { from week I to week I5. } \\
\text { Methotrexate group: ( } \mathrm{n}=1 \mathrm{I0}) \text {, received single weekly dose initiated at } 7.5 \mathrm{mg} \\
\text { per week, increased to a maximum of } 25 \mathrm{mg} \text { per week (depending on response) } \\
\text { Placebo: }(n=53)\end{array}$ \\
\hline $\begin{array}{l}\text { BELIEVEc }^{c} \\
(n=730)\end{array}$ & $\begin{array}{l}\text { Phase Illb randomized, double-blind, } \\
\text { vehicle-controlled study of adalimumab } \\
\text { with and without calcipotriol/betamethasone } \\
\text { topical treatment }\end{array}$ & $\begin{array}{l}\text { I6-week study } \\
\text { Adalimumab + calcipotriol/betamethasone group: }(n=366) 80 \mathrm{mg} \text { loading dose } \\
\text { of adalimumab at week } 0 \text { and then } 40 \mathrm{mg} \text { eow, calcipotriol/betamethasone was } \\
\text { applied daily for } 4 \text { weeks. } \\
\text { Adalimumab + drug-free vehicle: }(n=364) 80 \mathrm{mg} \text { loading dose of adalimumab at } \\
\text { week } 0 \text { and then } 40 \mathrm{mg} \text { eow; drug-free vehicle was applied daily for } 4 \text { weeks. }\end{array}$ \\
\hline
\end{tabular}

Notes: ${ }^{2}$ REVEAL ${ }^{12}$, Randomized Controlled Evaluation of Adalimumab every-other-week dosing in moderate to severe psoriasis trial; ${ }^{\text {}} \mathrm{CHAMPION}{ }^{16}$, Comparative study of Humira vs Methotrexate vs Placebo in Psoriasis patients; 'BELIEVE ${ }^{18}$, study of the efficacy and safety of adalimumab with and without calcipotriol/betamethasone topical treatment in patients with moderate to severe psoriasis.

Abbreviation: eow, every other week.

moderate to severe plaque psoriasis for at least 6 months, and stable plaque psoriasis for at least 2 months before screening. Prior treatment with topical, systemic, or phototherapy was acceptable after a washout period, and all patients were screened for latent tuberculosis upon entry. Patients were excluded if they were previously exposed to anti-TNF therapy, had poorly controlled medical conditions, or required systemic corticosteroids during the trial.

The trial spanned 52 weeks and had three time periods of assessments - 16 weeks, 33 weeks, and 52 weeks. Those who obtained a PASI score of 75 or greater or achieved a physician global assessment (PGA) score of clear or minimal disease continued onto the next period of the study. Period A (0-16 weeks) enrolled 1,212 patients; 814 received an $80 \mathrm{mg}$ adalimumab-loading dose at week 0 and then $40 \mathrm{mg}$ eow starting at week 1 , while 398 received placebo. In period B, 578 participants were continued on adalimumab $40 \mathrm{mg}$ eow, and 26 participants originally on placebo were started on $40 \mathrm{mg}$ eow after an $80 \mathrm{mg}$ loading dose. In period C, 490 participants who were originally given adalimumab in period A and continued through period B were split into two groups. In one group, 250 participants were continued on $40 \mathrm{mg}$ eow, and 240 were switched to placebo. Those who were on placebo in period $\mathrm{A}$ and began the drug in period $\mathrm{B}$ were continued on $40 \mathrm{mg}$ eow through period $\mathrm{C}$.

Adalimumab demonstrated significant improvement versus placebo in period A (0-16 weeks). A PASI score of 75 or greater was achieved in $71 \%$ of participants receiving adalimumab, versus $7 \%$ of participants on placebo. Furthermore, a PASI score of 90 was achieved in $45 \%$ of those on adalimumab in this time period, compared with $2 \%$ on placebo. A PGA score of clear or minimal disease was seen in $62 \%$ of participants on adalimumab, versus $4 \%$ of those receiving placebo.

In the third period of the study (period C), patients who had been receiving adalimumab from week 0 and maintained PASI scores of at least 75 were divided into two groups. One group continued receiving $40 \mathrm{mg}$ eow, while the other was reassigned to placebo. Of those who continued on adalimumab at week $33,79 \%$ maintained a PASI score of 75 , versus $43 \%$ of those who discontinued adalimumab and were re-assigned to placebo at week $33 .^{12}$

In a subsequent open-label extension study of retreatment with adalimumab, patients who completed REVEAL and other Phase II and III studies were eligible for enrollment. Out of an initial 1,468 patients receiving $40 \mathrm{mg}$ eow, those with a PGA of 3 or greater were not included in the open-label extension. 
Those with a PGA of 2 or less $(n=525)$ were withdrawn from treatment to assess time to relapse and response to retreatment. The withdrawal period lasted 40 weeks or until relapse (PGA of greater than or equal to 3). Of those withdrawn, 285 had stable psoriasis control and 178 relapsed, with a mean number of days to relapse of 141 days. All patients were given an initial loading dose of $80 \mathrm{mg}$ followed by $40 \mathrm{mg}$ eow for 16 weeks either at relapse or completion of the 40 weeks. The results of this study showed a significant response to adalimumab re-treatment in those who relapsed, with $29 \%, 54 \%, 62 \%$, and $69 \%$ of patients obtaining a PGA of clear or minimal at week 4, 8, 12, and 16, respectively. In those who did not relapse, $89 \%$ achieved PGA $0 / 1$, demonstrating that it is better to restart therapy before relapse. They also observed a more significant response to retreatment when patients were given a loading dose of $80 \mathrm{mg}$ then $40 \mathrm{mg}$ eow, versus restarting at $40 \mathrm{mg}$ eow. $^{13}$

In a 2011 study, researchers evaluated the efficacy of retreatment with adalimumab in moderate to severe psoriasis following treatment interruption after 33 weeks or $>3$ years of $40 \mathrm{mg}$ eow dosing. The study examined retreatment in two study populations - medium-term responders (treatment interrupted at 33 weeks) and longterm responders (treatment interrupted at 160 weeks). The medium-term responders were comprised of 202 patients who had PGA of 0 or 1 when treatment was interrupted, out of which 71 had PGA of at least 3 at end of treatment interruption and were subsequently retreated. The longterm responders were comprised of 103 patients with a PGA of 0 or 1 when treatment was interrupted, out of which 42 returned to a PGA of 3 or greater at the end of treatment interruption and were retreated. Only the long-term responders were blinded during treatment interruption, and they did not receive placebo. The long-term responders received an initial $80 \mathrm{mg}$ dose of adalimumab when treatment was reinitiated, whereas the medium-term responders were restarted with an initial $40 \mathrm{mg}$ dose. Duration of retreatment was 16 weeks for long-term and 24 weeks for medium-term responders. The study found that substantial efficacy was regained by both mediumand long-term responders, despite differences in duration of treatment prior to interruption, body surface area at initiation of treatment, blinding during treatment interruption, and whether an $80 \mathrm{mg}$ adalimumab starting dose was administered when retreatment began. ${ }^{14}$

A further evaluation of the REVEAL study evaluated safety and efficacy of adalimumab. Analysis of the data showed that adalimumab effectiveness was equal in both sexes, and strong responses were observed regardless of disease duration prior to treatment. It was also shown to be efficacious across all weight categories except for obese (body mass index $[\mathrm{BMI}]>30 \mathrm{~kg} / \mathrm{m}^{2}$ ) patients who demonstrated slightly less response rates $(65 \%$ response versus $79 \%$ response in BMI $<30 \mathrm{~kg} / \mathrm{m}^{2}$ ). The response was also slightly less in patients over 65 years old. A failure to respond to previous systemic therapy, including biologics, did not preclude patients from responding. ${ }^{15}$

The Comparative Study of Humira vs Methotrexate vs Placebo in Psoriasis Patients (CHAMPION) study was a randomized, double-blind, placebo-controlled comparative study of the safety and efficacy of adalimumab versus methotrexate versus placebo in patients with psoriasis. A total number of 271 patients with chronic plaque psoriasis were assigned into three groups. The adalimumab group $(\mathrm{n}=108)$ was administered an $80 \mathrm{mg}$ loading dose at week 0 , then $40 \mathrm{mg}$ eow from week 1 to week 15 . The oral methotrexate group $(n=110)$ received a single weekly dose initiated at $7.5 \mathrm{mg}$ per week and increased up to a maximum of $25 \mathrm{mg}$ per week depending on response. The placebo group was composed of the remaining 53 participants. The primary efficacy endpoint was the proportion of patients receiving at least a $75 \%$ improvement of PASI after 16 weeks. The efficacy endpoint was achieved by $79.6 \%$ of adalimumabtreated patients versus $35.5 \%$ of methotrexate-treated patients versus $18.9 \%$ of placebo-treated patients. Complete clearance of the disease was obtained in $16.7 \%$ of patients in the adalimumab group. The response to therapy was observed most rapidly in adalimumab-treated patients, with $57 \%$ improvement of PASI scores at week 4 . The study also looked at adverse events throughout treatment. The results showed similar adverse event rates across treatment groups, with the highest number of events leading to discontinuation in the methotrexate group. The methotrexate group experienced increased liver abnormalities compared with both adalimumab and placebo. ${ }^{16}$

In a subsequent benefit risk analysis, researchers used adverse event-free response days as a measurement of safety and efficacy amongst adalimumab, methotrexate, and placebo. They found adalimumab had the lowest number of adverse events as well as the highest number of adverse event-free response days. Patients treated with adalimumab experienced an average of 52.3 adverse event-free response days compared with 13.3 days for methotrexate and 10.4 days for placebo. They concluded that treatment with adalimumab was associated with a superior benefit-risk profile compared with methotrexate or placebo, and the advantage was statistically significant as early as the first 30 days. ${ }^{17}$ 
The addition of topical corticosteroids to adalimumab therapy was examined by the BELIEVE study: a Phase IIIb randomized, double-blind, vehicle-controlled study of adalimumab with and without calcipotriol/betamethasone topical treatment. The trial was conducted over a 16 -week period, with all patients receiving adalimumab $80 \mathrm{mg}$ loading dose followed by $40 \mathrm{mg}$ eow through week $1-15$ in addition to calcipotriol/betamethasone or drug-free vehicle applied once daily for 4 weeks. A total of 730 patients were enrolled in the study, with 366 receiving adalimumab + calcipotriol/betamethasone and 364 receiving adalimumab + drug-free vehicle. A PASI-75 response was originally higher in the combination therapy group, with $14.8 \%$ versus $5.8 \%$ in adalimumab + vehicle at week 2 . At week 4 , the PASI-75 was $70.7 \%$ for combination therapy versus $32.4 \%$ for adalimumab + vehicle. However, after week 4 , the trend was towards a higher response with adalimumab monotherapy. No statistical difference was noted in PASI-75 response at week 16 (64.8\% for adalimumab + calcipotriol/betamethasone versus $70.9 \%$ for adalimumab + vehicle). ${ }^{18}$

In a study reviewing the efficacy, safety, and medication cost implications of adalimumab, patients with suboptimal response to $40 \mathrm{mg}$ eow dosing were switched to a $40 \mathrm{mg}$ weekly dosing schedule. A total of 1,256 patients with moderate to severe psoriasis who had received blinded adalimumab $40 \mathrm{mg}$ eow or placebo in antecedent Phase II/III studies enrolled in an open-label extension study. Patients were continued on $40 \mathrm{mg}$ eow, except for those with a less than PASI-50 response who could increase dosage to $40 \mathrm{mg}$ weekly. The dose escalation of $40 \mathrm{mg}$ weekly was continued until patients achieved a PASI-75 response, then biweekly dosing was resumed. In total, $27.8 \%$ of patients underwent dosage escalation, and after 12 weeks, 26.6\% achieved substantial clinical improvement. Safety results were comparable between the eow dosing and weekly dosing groups. ${ }^{19}$

Of particular importance to prescribing clinicians, besides the safety and efficacy profile of adalimumab, is its success in terms of adherence to treatment regimens. A 2011 study found that up to $29 \%$ of patients discontinue the anti-TNF agents within a $24-30$-week treatment period. The study examined adherence to treatment of infliximab, adalimumab, and etanercept as an indicator of treatment success - taking into account the various predictors of drug survival. The most significant predictors were sex, the anti-TNF agent, and the previous response to an anti-TNF agent. The researchers observed the longest drug survival rate for infliximab followed by adalimumab with $25.5 \%$ versus $32 \%$ withdrawing from treatment, respectively. While no difference in the number of adverse events was found, efficacy was found to decrease over time, with infliximab being the longest drug survival at $70 \%$ adherence at 4 years, versus $40 \%$ for both adalimumab and etanercept. ${ }^{20}$

In a more recent study published in 2013, drug survival rates and PASI scores for patients treated with infliximab $(n=38)$, adalimumab $(n=59)$, and ustekinumab $(n=30)$ over a 12-month period were compared. In contrast to the previous study, adalimumab drug survival rate was found to be superior to infliximab, with $79.7 \%$ versus $73.3 \%$, respectively. Ustekinumab was found to have the highest drug survival rate, $96.7 \%$. The number of patients who achieved a PASI-75 score or greater were $68.4 \%, 50.8 \%$, and $63.3 \%$ for infliximab, adalimumab, and ustekinumab at 12 months, respectively. ${ }^{21}$

While a large percentage of patients with psoriasis are controlled by topicals and phototherapy, safety and efficacy of transitioning patients from one therapy to another is important in clinical practice. A 2011 study reviewed transitioning a patient to adalimumab following a suboptimal response to etanercept, methotrexate, or phototherapy. The primary endpoint was the percentage of patients achieving a PGA of "clear" or "minimal". In this 16-week, open-label, Phase IIIb trial, patients who previously had a suboptimal response to the treatments mentioned above experienced a 50\% likelihood of achieving a clinically relevant response to adalimumab. Patients were found to display a significant improvement in efficacy in as early as 4 weeks after transition. Adalimumab was determined to be efficacious regardless of age, sex, duration of disease, weight, baseline PGA, or duration of suboptimal treatment. However, when compared by substudy, men and patients with a shorter duration of psoriasis responded better to adalimumab when compared with women and those with a longer duration of disease. Researchers also found that transitioning patients to adalimumab was associated with a reduction in serum C-reactive protein concentrations. Patients had acceptable safety outcomes and a low risk of flare, despite immediate discontinuation of previous therapy and no dosage tapering or overlap. ${ }^{22}$

Several studies, including REVEAL, CHAMPION, and BELIEVE, all serve as evidence of adalimumab's efficacy in the treatment of chronic plaque psoriasis. However, there has been evidence of decreased efficacy in patients who develop anti-drug antibodies (ADAs). These ADAs were originally described in patients treated for rheumatoid arthritis but have subsequently been found in psoriasis patients. ${ }^{23}$ In a systematic review of 25 studies with a total of 7,969 psoriasis patients, antibodies specific to adalimumab were 
reported in $6.6 \%-44.8 \%$ of patients. The presence of antiadalimumab antibodies was associated with lower serum concentrations in $60 \%$ of the studies and reduced clinical efficacy in $80 \%$ of the studies reviewed. Similarly, a 2013 analysis of ADA formation in plaque psoriasis patients treated with adalimumab $(n=80)$, demonstrated ADA formation in $49 \%$ of patients. The data from this study was collected over a 1-year period; where $90 \%$ of the patients who were positive for ADA developed them within the first 24 weeks of treatment. In those patients, increasing eow to weekly dosing was less clinically efficacious. They concluded that the presence of ADA is correlated with drug concentration and clinical response. The development of ADAs poses a significant challenge to the long-term efficacy of biologic therapy. The concomitant use of methotrexate with anti-TNF agents to prevent ADA formation has been cited in other inflammatory diseases. ${ }^{24}$ A 2012 study reviewed adding various dosages of methotrexate to adalimumab therapy in rheumatoid arthritis patients. A total of 272 patients were placed in either no dose, low dose, intermediate dose, or high dose methotrexate therapy and followed over 154 weeks. The results showed a decrease in ADA formation with increased methotrexate dosage. ${ }^{25}$ Further exploration in this area is required in order to optimize the therapeutic response of adalimumab in psoriasis patients. ${ }^{26}$

\section{Safety}

Newly introduced pharmaceuticals are all subject to extensive safety data collection in trials, and research prior to and after they are available to the public. TNF antagonists, as immunemodulators, are especially important in regards to safety data collection, including adverse events and multiple safety registries both in the US and abroad. ${ }^{8}$ The safety package insert includes a black-box warning about the risk of pulmonary and extrapulmonary tuberculosis and the need for evaluation and screening of tuberculosis before and during treatment. Treatment for tuberculosis should be initiated prior to starting adalimumab therapy. There is also an increased risk of developing certain bacterial (Legionella, Listeria) and fungal (histoplasmosis) infections. The use of adalimumab in adolescents and children has been associated with lymphoma and other malignancies. In young adults with inflammatory bowel disease, there have been cases of fatal hepatosplenic T-cell lymphoma (specifically with concomitant use of azathioprine or mercaptopurine). Adalimumab use in pregnancy is considered category B: no evidence of harm to the fetus was found in studies on monkeys; however, no well controlled studies have been performed in pregnant women. ${ }^{10}$ The most frequent reported adverse events in clinical trials were nasopharyngitis and upper respiratory tract infection. Injection-site reaction appears to be transient and reported as mild to moderate; occurring in less than $5 \%$ of treated patients. ${ }^{11}$

The long-term safety of adalimumab treatment in moderate to severe psoriasis was reviewed in 2011. This analysis examined safety data among various datasets, including an all adalimumab treatment (AAT) population, an everyother-week population, and placebo-controlled portions of clinical trials. For the AAT population, cumulative data were reviewed from 13 clinical trials and over 7,000 patients. More specifically, methods for this analysis included data from four Phase II, three Phase II/III, two Phase III, three Phase IIIb, and one Phase IV studies. In the every-other-week population, longitudinal data were collected from 1,403 patients receiving adalimumab $40 \mathrm{mg}$ eow. The placebocontrolled dataset was comprised of 572 patients, with a total of 173 patient-years (PYs) of exposure to placebo. ${ }^{27}$

In $\geq 5 \%$ of patients, adverse events that occurred were nasopharyngitis, upper respiratory infection, and headache in both the AAT population and the every-other-week population. There were eight deaths as of November 2009, one of those deaths was attributed to two adverse events, which caused the number of fatal adverse events (9) to exceed the number of deaths (8). In this analysis, two fatal adverse events were due to myocardial infarctions, two were due to coronary artery disease, two to cerebral vascular accidents, one to an aortic aneurysm, one to suicide, and one to an unknown cause.

This integrated analysis of data with greater than 4,350 PYs of adalimumab exposure showed that rates of serious adverse events, serious infections, and malignancies in patients were low, and most adverse events decreased with increased exposure to drug. Results proved that the incidence rates of treatment-emergent adverse events were generally low and stable, consistent with a favorable benefit-risk profile.

Similarly, in a 2010 review of the long-term clinical trial safety experience with adalimumab $40 \mathrm{mg}$ eow for moderate to severe psoriasis, adalimumab was found to have a favorable safety profile. This analysis used data from 1,403 moderate to severe psoriasis patients with $>2,800 \mathrm{PYs}$ of exposure to adalimumab. The results indicated that incidence rates of treatment-emergent adverse events were generally low and stable with increased, long-term exposure. Rates of treatmentemergent serious adverse events, serious infections, and malignancies were low, and most serious adverse event rates were stable or declined with continued adalimumab exposure in a patient population with a mean duration of exposure of 2 years and up to 5.7 years. $^{28}$ 
The effectiveness and safety of adalimumab has been examined in several Phase III clinical trials and the open-label extension study; however, there is also an ongoing Phase IV observational registry study termed ESPRIT. ${ }^{28}$ The ESPRIT is a 10 -year, prospective, multicenter, international registry study of the long-term safety and effectiveness of adalimumab as used in routine clinical practice in adult patients with chronic moderate to severe plaque psoriasis. While the study is currently ongoing, 4-year interim data have been recently published. As of 2013, a total of 6,040 patients in the all-Rx population and a subset of 2,573 patients in the new-Rx population are in the registry. Out of these, $93 \%$ continue in the registry during this 4 -year period. For the all-Rx population, incidence rates for serious infections and malignancies (Table 2) were 1.2 events/100 PYs and 1.1 events/100 PYs, respectively. Incidence rates of serious infection and malignancies remained stable compared with the 3 -year interim report. The percentage of patients experiencing effective treatment with adalimumab, as evidence by PGA of "clear or minimal", increased from $56.8 \%$ at 12 months to $59 \%$ at 24 months, $60.1 \%$ at 36 months, and $63.2 \%$ at 42 months. The ongoing data collection in the ESPRIT registry will provide valuable information about the safety and efficacy of adalimumab therapy in psoriasis. ${ }^{28}$

\section{Patient-reported outcomes}

While the ESPRIT registry and other long-term data provide valuable information about the therapeutic safety and efficacy of adalimumab, it is also important to examine

Table 2 Adverse events reported in ESPRIT data registry with rate $\geq 0.1$ events/100 PYs in the all-Rx population

\begin{tabular}{|c|c|c|c|c|}
\hline \multirow[t]{3}{*}{ System organ class } & \multicolumn{4}{|c|}{ All-Rx $N=6,040$} \\
\hline & \multirow[t]{2}{*}{$\mathbf{N}$} & \multirow[t]{2}{*}{$\%$} & \multicolumn{2}{|c|}{$\begin{array}{l}\text { PYs of obs duration } \\
n=12,400.9\end{array}$} \\
\hline & & & Events & E/I00 PYs \\
\hline Any SAE & 419 & 6.9 & 694 & 5.6 \\
\hline Cardiac disorders & 51 & 0.8 & 64 & 0.5 \\
\hline Infections & 11 & 1.9 & 149 & 1.2 \\
\hline Abscesses & 11 & 0.2 & 12 & $<0.1$ \\
\hline Cellulitis & 25 & 0.4 & 29 & 0.2 \\
\hline Pneumonia & 18 & 0.3 & 18 & 0.1 \\
\hline TB & 3 & $<0.1$ & 3 & $<0.1$ \\
\hline $\begin{array}{l}\text { Neoplasm benign, malignant, } \\
\text { and unspecified }\end{array}$ & 76 & 1.3 & 90 & 0.7 \\
\hline SCC & 9 & 0.1 & 18 & 0.1 \\
\hline Nervous system disorders & 40 & 0.7 & 49 & 0.4 \\
\hline Skin and tissue disorders & 17 & 0.3 & 20 & 0.2 \\
\hline Psoriasis & 12 & 0.2 & 15 & 0.1 \\
\hline
\end{tabular}

Abbreviations: PY, patient-year; SAE, serious adverse event; TB, tuberculosis; SCC, squamous cell carcinoma; obs, observation. patient-reported outcomes. In numerous studies, patientreported outcomes were evaluated as a secondary endpoint using measures such as the Dermatology Life Quality Index (DLQI), the Short-Form 36 Health Survey (SF-36), the Work Productivity and Activity Impairment Questionnaire Specific Health Problem, and several other patient-reported symptoms scales. Results demonstrated that the adalimumabtreated groups reported significantly greater improvements in DLQI total score, SF-36 Physical Component Summary score, and Mental Component Summary scores. Adalimumab has been shown to be efficacious in improving dermatologyspecific and general health-related quality of life, work, and activity limitations, and psoriasis-related symptoms in patients with moderate to severe psoriasis. ${ }^{29}$ Similarly, in a 12-week, Phase II, randomized controlled trial, patients displayed significantly greater improvements versus placebo patients in areas such as social functioning, mental health domains, bodily pain, and vitality. ${ }^{30}$ The degree of sleep impairment and work productivity was assessed in a 16-week, open-label, Phase IIIb progress trial, revealing that treatment with adalimumab significantly improved sleep quality by $15 \%$ from baseline, which led to other patient-reported outcomes including increased health-related quality of life, work productivity, daily activity, and disease-related pain. ${ }^{31}$

\section{Conclusion}

Adalimumab was introduced in 2002 and was approved for use in the treatment of chronic plaque psoriasis in 2008. Since then, it has proven to be very efficacious in the clinical setting; with reports of $79.6 \%$ of patients showing a PASI of 75 as early as 16 weeks. ${ }^{16}$ Safety outcomes have been favorable and demonstrate low rates of serious adverse events and infections, and a positive risk-benefit profile. As data collection continues in the post-marketing Phase IV ESPRIT registry, more long-term information about the safety and efficacy of adalimumab will become available. Further research is needed to examine the effect of antidrug antibodies on the drug's clinical effectiveness and the potential for using concomitant therapies to reduce their development. Additionally, an analysis directly comparing the various biologic therapies available for psoriasis would provide invaluable information to the existing literature. Another important area of research will be the effect of adalimumab (and other anti-TNF drugs) on the psoriatic comorbid conditions.

\section{Disclosure}

Dr Francisco Kerdel has participated in advisory boards, speaking engagements, and has received grants for clinical 
studies from AbbVie (previously Abbott) Pharmaceuticals. There are no other conflicts of interest to report.

\section{References}

1. Van der Koff P, Schalkwijk J. Psoriasis. In: Bolognia JL, Jorizzo JL, Rapini RP, et al. Dermatology. 2nd ed. Spain: Mosby Elsevier; 2008: $115-135$.

2. Andressen $\mathrm{C}$, Henseler T. Inheritance of psoriasis: analysis of 2035 family histories. Hautarzt. 1982;33:214-217.

3. Kremers HM, McEvoy MT, Dann FJ, et al. Heart disease in psoriasis. J Am Acad Dermatol. 2007;57:347-354.

4. Schmitt JM, Ford DE. Role of depression in quality of life for patients with psoriasis. Dermatology. 2007;215:17-27.

5. National Psoriasis Foundation. Statistics. Available from: http://www. psoriasis.org/about/stats. Accessed December 21, 2013.

6. Schon MP, Boehncke WH. Psoriasis. N Eng J Med. 2005;352: 1899-1912.

7. Krueger JG, Bowcock A. Psoriasis pathophysiology: current concepts of pathogenesis. Ann Rheum Dis. 2005;64(Suppl 2):ii30-ii36.

8. Tracey D, Klareskog L, Sasso EH, et al. Tumor necrosis factor antagonist mechanism of action: a comprehensive review. Pharmacol Ther. 2008;117:244-279.

9. Schmitt J, Wozel G. Targeted treatment of psoriasis with adalimumab: a critical appraisal based on a systematic review of the literature. Biologics. 2009;3:303-318.

10. Food and Drug Administration. Humira (adalimumab) [package insert]. US Govt Lic No 0043, Abbott Laboratories. Available from: http://www. accessdata.fda.gov/drugsatfda_docs/label/2011/125057s0276lbl.pdf. Accessed January 4, 2014.

11. Gordon KB, Langley RG, Leonardi C, et al. Clinical response to adalimumab treatment in patients with moderate to severe psoriasis: double-blinded, randomized controlled trial and open-label extension study. J Am Acad Dermatol. 2006;55:598-606.

12. Menter A, Tyring SK, Gordon K, et al. Adalimumab therapy for moderate to severe psoriasis: a randomized, controlled Phase III trial. J Am Acad Dermatol. 2008;58:106-115.

13. Papp K, Crowley J, Ortonne JP, et al. Adalimumab for moderate to severe chronic plaque psoriasis: efficacy and safety of retreatment and disease recurrence following withdrawal from therapy. Br J Dermatol. 2011;164:434-441.

14. Crowley J, Ortonne JP, Gu Y, et al. The efficacy of retreatment with adalimumab in moderate to severe psoriasis following treatment interruption after 33 weeks or $>3$ years of $40 \mathrm{mg}$ every other week dosing. J Am Acad Dermatol. 2011;64:2 Suppl AB151.

15. Menter A, Gordon K, Leonardi C, et al. Efficacy and safety of adalimumab across subgroups of patients with moderate to severe psoriasis. J Am Acad Dermatol. 2010;63:448-456.

16. Saurat J, Stingl G, Dubertret L, et al. Efficacy and safety results from the randomized controlled comparative study of adalimumab vs methotrexate vs placebo in patients with psoriasis (CHAMPION). Br J Dermatol. 2008;158:558-566.

17. Reich K, Signorovitch J, Ramakrishnan K, et al. Benefit-risk analysis of adalimumab versus methotrexate and placebo in the treatement of moderate to severe psoriasis; comparison of adverse-event free response days in the CHAMPION trial. J Am Acad Dermatol. 2010;63:1011-1018.
18. Thaci D, Ortonne JP, Chimenti S, et al. A Phase IIIb, multicentre, randomized, double-blind, vehicle-controlled study of the efficacy and safety of adalimumab with and without calcipotriol/betamethasone topical treatment in patients with moderate to severe psoriasis: the BELIEVE study. Br J Dermatol. 2010;163:402-411.

19. Leonardi C, Sobell JM, Crowley JJ, et al. Efficacy, safety, and medication cost implications of adalimumab $40 \mathrm{mg}$ weekly dosing in patients with psoriasis with suboptimal response to $40 \mathrm{mg}$ every other week dosing: results from an open-label study. Br J Dermatol. 2012;167: 658-667.

20. Gniadecki R, Kragballe K, Damn TN, et al. Comparison of drug survival rates for adalimumab, etanercept and infliximab in patients with psoriasis vulgaris. Br J Dermatol. 2011;164:1091-1096.

21. Umezawa Y, Nobeyama Y, Hayashi M, et al. Drug survival rates in patients with psoriasis after treatment with biologics. $J$ Dermatol. 2013;40:1008-1013.

22. Strober BE, Poulin Y, Kerdel FA, et al. Switching to adalimumab for psoriasis patients with a suboptimal response to etanercept, methotrexate, or phototherapy: efficacy and safety results from an open-label study. J Am Acad Dermatol. 2011;64:671-681.

23. Ordas I, Feagan BG, Sandborn WJ. Therapeutic drug monitoring of tumor necrosis factor antagonists in inflammatory bowel disease. Clin Gastroenterol Hepatol. 2012;10:1079-1087.

24. Krieckaert CL, Bartelds GM, Lems WF, et al. The effect of immunomodulators on the immunogenicity of TNF-blocking therapeutic monoclonal antibodies: a review. Arthritis Res Ther. 2010;12:217.

25. Krieckaert CL, Nurmohamed MT, Wolbink GJ. Methotrexate reduces immunogenicity in adalimumab treated rheumatoid arthritis patients in a dose dependent manner. Ann Rheum Dis. 2012;71:1914-1915.

26. Hsu L, Snodgrass BT, Armstrong AW. Anti-drug antibodies in psoriasis: a systematic review. Br J Dermatol. Epub October 1, 2013.

27. Leonardi C, Papp K, Strober B, et al. The long-term safety of adalimumab treatment in moderate to severe psoriasis: a comprehensive analysis of all adalimumab exposure in all clinical trials. Am J Clin Dermatolog. 2011;12:321-337.

28. Thaci D, Kerdel F, Wu J. Fourth-year interim safety and patient outcomes from ESPRIT, a 10-year postmarketing surveillance registry of adalimumab for moderate to severe psoriasis. Presented at: The 14th Annual Las Vegas Dermatology Seminar and presented at the 10th Annual SDEF Psoriasis Forum; October 31-November 2, 2013; Las Vegas, NV.

29. Revicki DA, Willian MK, Menter A, et al. Impact of adalimumab treatment on patient-reported outcomes: results from a Phase III clinical trial in patients with moderate to severe plaque psoriasis. J Dermatolog Treat. 2007;18:341-350.

30. Revicki DA, Menter A, Feldman S, et al. Adalimumab improves health-related quality of life in patients with moderate to severe plaque psoriasis compared with the United States general population norms: results from a randomized, controlled Phase III study. Health Qual Life Outcomes. 2008;6:75.

31. Stober BE, Sobell JM, Duffin KC, et al. Sleep quality and other patient-reported outcomes improve after patients with psoriasis with suboptimal response to other systemic therapies are switched to adalimumab: results from PROGRESS, an open-label Phase IIIB trial. Br J Dermatol. 2012;167(6):1374-1381.
Psoriasis: Targets and Therapy

\section{Publish your work in this journal}

Psoriasis: Targets and Therapy is international, peer-reviewed, open access journal focusing on psoriasis, nail psoriasis, psoriatic arthritis and related conditions, identification of therapeutic targets and the optimal use of integrated treatment interventions to achieve improved outcomes and quality of life. The manuscript management system

\section{Dovepress}

is completely online and includes a very quick and fair peer-review system. Visit http://www.dovepress.com/testimonials.php to read real quotes from published authors. 